Review began 01/26/2022 Review ended 03/03/2022 Published 03/06/2022

(c) Copyright 2022

Prabhu et al. This is an open access article distributed under the terms of the Creative Commons Attribution License CC-BY 4.0., which permits unrestricted use, distribution, and reproduction in any medium, provided the original author and source are credited.

\title{
Urinary Albumin to Creatinine Ratio to Predict Diabetic Retinopathy: The Eyes Have It!
}

\author{
Divya Prabhu ${ }^{1}$, Akshay Rao ${ }^{2}$, Anjana Rajanna ${ }^{1}$, Sakthi Kannan ${ }^{1}$, Selva Kumar ${ }^{1}$ \\ 1. Internal Medicine, Ramaiah Medical College, Bangalore, IND 2. Internal medicine, Ramaiah Medical College, \\ Bangalore, IND
}

Corresponding author: Akshay Rao, akshayrao19@yahoo.com

\section{Abstract \\ Introduction}

The Coronavirus disease 2019 (COVID-19) pandemic has provided a push in the search for alternative screening methods to replace annual fundoscopic examination of patients with type 2 diabetes mellitus (T2DM) to detect diabetic retinopathy (DR).

\section{Materials and methods}

This retrospective study was conducted using the data of T2DM patients from their routine follow-up hospital visits. The details from their history and physical examination were extracted. As part of their routine follow-up visit, they had undergone a panel of investigations that included blood glucose measurements and urinary albumin excretion measurements. Univariate and logistic multivariate regression analyses were applied to identify the potential clinical and laboratory parameters associated with the presence of DR in them.

\section{Results}

Analysis of the medical records of 272 T2DM patients revealed that 147 patients had DR while 125 did not. Furthermore, 135 had non-proliferative DR (64 mild, 53 moderate, and 18 severe grades), whereas the remaining 12 had proliferative $\mathrm{DR}$. On sequential univariate and multiple regression analysis, urinary albumin creatinine ratio (UACR), known duration of T2DM, and history of ischemic heart disease were seen to be independently associated with the presence of DR. Median UACR for those without DR was 42.6 $\mathrm{mg} / \mathrm{g}$ (range $18.21-183.3 \mathrm{mg} / \mathrm{g}$ ) while for those with retinopathy it was $214 \mathrm{mg} / \mathrm{g}$ (range $45.4-1260 \mathrm{mg} / \mathrm{g}$ ) $(p<0.001)$. The receiver operating characteristics curve analysis provided an area under the curve of $70 \%$ for UACR. UACR value of $140 \mathrm{mg} / \mathrm{g}$ could predict the presence of DR with a sensitivity of $60.5 \%$ \& specificity of $72 \%$, as well as had positive and negative likelihood ratios of 2.16 and 0.54 , respectively.

\section{Conclusion}

UACR has the potential to be used as a screening tool for DR until the easing of social restrictions due to the COVID-19 pandemic.

Categories: Endocrinology/Diabetes/Metabolism, Internal Medicine, Ophthalmology

Keywords: macroalbuminuria, microalbuminuria, retinopathy screening, fundoscopy, diabetic retniopathy, uacr

\section{Introduction}

Type 2 diabetes mellitus (T2DM) is characterized by microvascular damage mediated by long-standing hyperglycemia. Microvascular complications like diabetic retinopathy (DR), nephropathy, and neuropathy tend to occur as a result of poorly controlled T2DM. These complications contribute greatly to the morbidity and mortality in T2DM. DR is increasingly being recognized as a significant cause of blindness in India as in the rest of the world [1,2]. In advanced stages, it can progress in a relentless manner despite best attempts to achieve optimal blood glucose control [3]. It has been recommended that all patients with T2DM undergo fundoscopic examination at diagnosis and at least annually thereafter for early detection and management of DR [4].

The pandemic of Coronavirus Disease 2019 (COVID-19) has disrupted this practice of routine fundoscopic examination [5]. Initially, the lockdown imposed by the government and subsequently the fear of COVID-19 exposure at hospitals may have deterred patients from adhering to their ophthalmologist appointment. In the midst of the deadly pandemic, when the healthcare services were stretched to their limits, screening of asymptomatic patients for retinal findings was accorded a lower priority. During fundus examination, the patient and the examiner are separated by virtually no distance. The risk of close contact with patients carrying potential subclinical COVID-19 infection might have also led to some degree of reluctance among the medical fraternity to perform fundoscopy for retinopathy screening. Indeed it has been acknowledged 
that ophthalmologists are at a higher risk of being infected with COVID-19 [6]. Consequently, this has spurred the search for alternative markers that can be used to screen T2DM patients for the need to undergo fundus examination.

The association between diabetic nephropathy and retinopathy in T2DM is not as strong as in the case of type 1 diabetes. This might be due to the presence of other contributors to renal injury in T2DM, such as associated essential hypertension, dyslipidemia, and atherosclerosis [7]. Yet one underlying mechanism that is common to the pathogenesis of both DR and diabetic nephropathy is endothelial dysfunction [8]. The histopathological hallmark of DR is a loss of pericytes which are cells that cover the retinal capillary endothelial cells and help to maintain the capillary tone. This is followed by thickening of the basement membrane and enhanced endothelial permeability. Identical histological changes are known to occur in the kidney within the glomeruli of nephrons which are the tuft of capillaries enclosed by the Bowman's capsule. This leads to an increase in endothelial permeability resulting in albuminuria $[9,10]$.

The determination of the urinary albumin to creatinine ratio (UACR) using spot, first-morning urine sample is a simple and accurate test to quantify albuminuria [11]. All patients with T2DM are recommended to test UACR levels annually in order to detect the occurrence of nephropathy [4]. Hence this non-invasive and widely available investigation has the potential to be used as an alternative to a fundoscopic screening of DR. This study aimed to determine the association of UACR with the presence of DR in patients with T2DM.

\section{Materials And Methods}

This retrospective study analyzed the data of patients who had visited the general medicine and ophthalmology out-patient departments (OPD) at a tertiary care hospital in South India between 2015 and 2018 for a regular follow-up of glycemic control. As part of their routine visit, all patients had undergone clinical assessment, biochemical and ophthalmological evaluation on an appointment basis.

Data, including the clinical history, known duration of T2DM, the presence of other non-communicable diseases, and medication details, was extracted from the medical records of all the patients. Educational qualifications and the details of physical activity recorded as per the global physical activity questionnaire were retrieved [12]. Physical examination details, including the values of their clinic blood pressure, height in meters $(\mathrm{m})$, and body weight in kilograms $(\mathrm{kg})$, was extracted. The body mass index (BMI) was derived using the formula BMI = weight $(\mathrm{kg}) /\{\text { height }(\mathrm{m})\}^{2}$. The fundoscopy findings were noted in accordance with the Early Treatment for Diabetic Retinopathy Study (ETDRS) classification to divide the patients into one out of five categories [13]:

i) No DR

ii) Mild non-proliferative diabetic retinopathy (NPDR): at least one microaneurysms; criteria not met for other levels of DR

iii) Moderate NPDR: microaneurysms or hemorrhages along with soft exudates, venous beading, and intraretinal microvascular abnormalities

iv) Severe NPDR: microaneurysms or hemorrhages in all four quadrants, venous beading in two or more quadrants, intraretinal microvascular abnormalities in at least one quadrant

v) Proliferative diabetic retinopathy (PDR): neovascularization and/or vitreous or pre-retinal hemorrhage.

The reports of laboratory tests performed during the same OPD visit were extracted, including the values of fasting (FBS) and post-prandial blood glucose (PPBS) by the hexokinase method, glycated hemoglobin (HbA1) by high-performance liquid chromatography, and serum creatinine using Jaffe kinetic method. The estimated glomerular filtration rate (eGFR) was calculated using the Chronic Kidney Disease Epidemiology Collaboration (CKD-EPI) formula [14]. For women, creatinine $<0.7 \mathrm{mg} / \mathrm{dl}$ eGFR $=144 \times($ creatinine/0.7) -0.329 $\times(0.993)$ age, and creatinine $>0.7 \mathrm{mg} / \mathrm{dl} \mathrm{eGFR}=144 \times($ creatinine $/ 0.7)-1.209 \times(0.993)$ age; and for men, creatinine $>0.9 \mathrm{mg} / \mathrm{dl} \mathrm{eGFR}=141 \times($ creatinine $/ 0.9)-1.209 \times(0.993)$ age.

The levels of albumin (automated turbidometry method) and creatinine (Jaffe kinase method) in the spot first-morning urine sample of the day of their visit that had been used to determine the UACR in the same visit was also extracted. Microalbuminuria was defined as a UACR between 30-300 mg/g and macroalbuminuria as UACR $>300 \mathrm{mg} / \mathrm{g}[15]$.

All patients with T2DM over the age of 18 years were included in this study. Patients excluded from the study were those who had any of the following to be documented in them: past medical history of non-diabetic kidney disease; a history of concurrent fever, urinary tract infection or were detected to have urinary calculi at the time of assessment; microscopic or macroscopic hematuria; or corneal opacities, hyper-mature cataracts or other illnesses interfering with the interpretation of the direct ophthalmology findings. 


\section{Statistical analysis}

Continuous data was represented as mean and standard deviation. Categorical data was represented in the form of frequencies and proportions. Data was entered into Microsoft Excel (Microsoft, Redmond, Washington) datasheet, and Statistical Package for Social Sciences version 20.0 (IBM Inc., Armonk, New York) was used to analyze the data. The Chi-square test or Fischer's exact test (for $2 \times 2$ tables only) was used as a test of significance for qualitative data. An independent $t$-test was used as a test of significance to identify the mean difference between two quantitative variables. ANOVA was used as a test of significance to identify the mean difference between more than two quantitative variables. Following a univariate analysis of the predictor variables, a logistic multiple regression analysis was performed to identify the variables associated independently with the presence of diabetic retinopathy. The receiver operating characteristic (ROC) curve was plotted for the UACR values, and optimal cut-off points were chosen for the calculation of sensitivity, specificity, positive and negative predictive values in order to predict the association with diabetic retinopathy. An AUC of 0.5 would mean no discrimination, 0.7 to 0.8 would be considered to be acceptable, 0.8 to 0.9 considered excellent, and greater than 0.9 would be considered outstanding. a p-value of $\leqslant 0.05$ was deemed to be statistically significant [16].

\section{Results}

The medical records of 300 consecutive patients with T2DM who visited the general medicine OPD between 2015 and 2018 were accessed. Owing to the presence of one or more exclusion criteria, 28 were not considered for inclusion in the study. Finally, the data from a total of 272 patients was analyzed for this study. This included 156 male and 116 female patients. The average age of the patients included in the study was $60.35 \pm 10.56$ years.

A total of $147 / 272$ patients were diagnosed to have diabetic retinopathy, whereas the rest 125 had no retinopathy on fundus examination during the OPD visit. Among those with retinopathy, 135 patients were found to have NPDR ( 64 patients had mild, 53 patients had moderate, and 18 patients had severe), whereas 12 patients were diagnosed with PDR.

An incremental trend was seen with respect to the mean age $(\mathrm{p}<0.001)$ as well as the median duration of diabetes $(\mathrm{p}<0.001)$ among patients having DR (Table 1). On univariate analysis, a significantly higher number of retinopathy patients were found to have associated comorbidities such as hypertension, coronary artery disease (CAD), and hypothyroidism in comparison to those diabetics without retinopathy. The differences in their current pattern of antidiabetic medications and their physical activity levels were also found to be significant. There was also a statistically significant difference noticed with regards to the history of the presence of T2DM among immediate family members between the two groups.

\begin{tabular}{|c|c|c|c|c|c|c|}
\hline & $\begin{array}{l}\text { No DR } \\
(\mathrm{N}=125)\end{array}$ & $\begin{array}{l}\text { Mild NPDR } \\
(\mathrm{N}=64)\end{array}$ & $\begin{array}{l}\text { Moderate NPDR } \\
(\mathrm{N}=53)\end{array}$ & $\begin{array}{l}\text { Severe NPDR } \\
(\mathrm{N}=18)\end{array}$ & PDR (N=12) & $\begin{array}{l}\mathrm{p} \text { - } \\
\text { value }\end{array}$ \\
\hline Age $^{*}$ (years) & $54.97 \pm 9.55$ & $61.06 \pm 8.69$ & $67.25 \pm 8.68$ & $69.06 \pm 7.32$ & $69.08 \pm 8.77$ & $<0.001$ \\
\hline $\begin{array}{l}\text { Duration of DM } \\
\text { (years) }\end{array}$ & $5(2.5-8.5)$ & $10(6-12)$ & 15(10-21.5) & 19(12-22.25) & 20(15.3-23) & $<0.001$ \\
\hline BMI $\left(\mathrm{Kg} / \mathrm{m}^{2}\right)$ & $\begin{array}{l}27.34(25.4- \\
29.5)\end{array}$ & 28.69(26.8-30.4) & 28.2(25.9-30.74) & $28.775(24.4-31.58)$ & $\begin{array}{l}30.18(24.08- \\
32.29)\end{array}$ & 0.125 \\
\hline \multicolumn{7}{|l|}{ Gender } \\
\hline Female & $58(46.4 \%)$ & $23(35.9 \%)$ & $21(39.6 \%)$ & $7(38.9 \%)$ & $58(46.4 \%)$ & \multirow{2}{*}{0.492} \\
\hline Male & $67(53.6 \%)$ & $41(64.1 \%)$ & $32(60.4 \%)$ & $11(61.1 \%)$ & 67(53.6\%) & \\
\hline \multicolumn{7}{|l|}{ Comorbidities } \\
\hline Hypertension & $44(35.2 \%)$ & $34(53.1 \%)$ & $33(62.3 \%)$ & $16(88.9 \%)$ & $8(66.7 \%)$ & $<0.001$ \\
\hline $\begin{array}{l}\text { Coronary artery } \\
\text { disease }\end{array}$ & $7(5.6 \%)$ & $8(12.5 \%)$ & $21(39.6 \%)$ & $8(44.4 \%)$ & $6(50.0 \%)$ & $<0.001$ \\
\hline Hypothyroid & 18(14.4\%) & $1(1.6 \%)$ & $4(7.5 \%)$ & $0(0 \%)$ & $0(0 \%)$ & 0.014 \\
\hline Family History of DM & $48(38.4 \%)$ & 34(53.1\%) & $26(49.1 \%)$ & $8(44.4 \%)$ & $9(75.0 \%)$ & 0.075 \\
\hline \multicolumn{7}{|l|}{ Education } \\
\hline Illiterate & $22(17.6 \%)$ & $14(21.9 \%)$ & $11(20.8 \%)$ & $2(11.1 \%)$ & $22(17.6 \%)$ & \\
\hline Schooling & $29(23.2 \%)$ & $15(23.4 \%)$ & $14(26.4 \%)$ & $8(44.4 \%)$ & $29(23.2 \%)$ & \\
\hline
\end{tabular}




\section{Cureus}

\begin{tabular}{|c|c|c|c|c|c|c|}
\hline PUC & $12(9.6 \%)$ & $4(6.2 \%)$ & $6(11.3 \%)$ & $0(0 \%)$ & $12(9.6 \%)$ & \multirow[t]{3}{*}{0.589} \\
\hline Graduation & $52(41.6 \%)$ & $24(37.5 \%)$ & 19(35.8\%) & $7(38.9 \%)$ & $52(41.6 \%)$ & \\
\hline Masters & $10(8.0 \%)$ & $7(10.9 \%)$ & $3(5.7 \%)$ & $1(5.6 \%)$ & $10(8.0 \%)$ & \\
\hline \multicolumn{7}{|l|}{ Current treatment } \\
\hline Not on treatment & $2(1.6 \%)$ & $0(0 \%)$ & $0(0 \%)$ & $0(0 \%)$ & $0(0 \%)$ & \multirow{4}{*}{0.04} \\
\hline Insulin & $78(62.4 \%)$ & $30(46.9 \%)$ & $20(37.7 \%)$ & 6(33.3\%) & $3(25.0 \%)$ & \\
\hline OAD & $14(11.2 \%)$ & $11(17.2 \%)$ & $8(15.1 \%)$ & $3(16.7 \%)$ & $6(50.0 \%)$ & \\
\hline Insulin and OAD & $31(24.8 \%)$ & 23(35.9\%) & $25(47.2 \%)$ & $9(50.0 \%)$ & $3(25.0 \%)$ & \\
\hline \multicolumn{7}{|l|}{ Activity } \\
\hline Inactive & $91(72.8 \%)$ & $42(66.7 \%)$ & $46(86.8 \%)$ & 17(94.4\%) & $9(75.0 \%)$ & \multirow{2}{*}{0.035} \\
\hline Active & $34(27.2 \%)$ & 21(33.3\%) & $7(13.2 \%)$ & $1(5.6 \%)$ & $3(25.0 \%)$ & \\
\hline FBS (mg/dl) & 182(161.5-216) & 185(156-214.75) & 184(152-216) & 213.5(166.5-218.25) & $\begin{array}{l}162(136.5- \\
213.15)\end{array}$ & 0.476 \\
\hline PPBS (mg/dl) & 232(202-288.5) & 232(202.5-283) & 233(207-290) & 274(225.75-329.5) & $\begin{array}{l}220(177.75- \\
313.5)\end{array}$ & 0.267 \\
\hline HbA1C (\%) & $9.5(7.8-11.2)$ & $9.25(7.8-11.05)$ & $9.7(7.9-11.5)$ & 10.8(9-12.9) & $9.6(7.225-10.125)$ & 0.115 \\
\hline $\begin{array}{l}\text { eGFR* }(\mathrm{ml} / \mathrm{min} / 1.73 \\
\left.\mathrm{m}^{2}\right)\end{array}$ & $74.95 \pm 26.09$ & $56.06 \pm 24.20$ & $56.90 \pm 29.41$ & $49.33 \pm 32.78$ & $54.41 \pm 43.12$ & $<0.001$ \\
\hline Uncontrolled FBS & 13(48.1\%) & $8(29.6 \%)$ & $3(11.1 \%)$ & $1(3.7 \%)$ & $2(7.4 \%)$ & 0.631 \\
\hline Uncontrolled PPBS & 13(43.3\%) & $9(30.0 \%)$ & $5(16.7 \%)$ & $0(0 \%)$ & $3(10.0 \%)$ & 0.248 \\
\hline Dyslipidemia & $36(38.7 \%)$ & $21(22.6 \%)$ & $25(26.9 \%)$ & $8(8.6 \%)$ & $3(3.2 \%)$ & 0.14 \\
\hline Microalbuminuria & $56(64.4 \%)$ & 16(18.4\%) & $12(13.8 \%)$ & $1(1.1 \%)$ & $2(2.3 \%)$ & $<0.001$ \\
\hline
\end{tabular}

\section{TABLE 1: Univariate analysis of variables for association with DR}

${ }^{*}$ Age and eGFR presented as mean \pm standard deviation, other variables are presented as median(inter-quartile range)

NPDR - non-proliferative diabetic retinopathy, PDR - proliferative diabetic retinopathy, DM - diabetes mellitus, BMI - body mass index, eGFR - estimated glomerular filtration rate, FBS - fasting blood sugar, PPBS - post-prandial blood glucose, HbA1c - glycated hemoglobin, UACR - urinary albumin to creatinine ratio, PUC - pre-university degree, OAD - oral antidiabetic medication

The UACR was found to be higher, and eGFR values were lower among those with DR. The median UACR value among patients with DR was found to be 214 (45.4-1260) $\mathrm{mg} / \mathrm{g}$, which was seen to be significantly higher than for those without retinopathy for whom it was $42.6 \mathrm{mg} / \mathrm{g}(18.21-183.3 \mathrm{mg} / \mathrm{g})(\mathrm{p}<0.001)$ (Table 2).

\begin{tabular}{|l|l|l|}
\hline & No DR $(\mathbf{m g} / \mathbf{g})$ & DR $(\mathbf{m g} / \mathbf{g})$ \\
\hline UACR median(interquartile range) & $42.6(18.21-183.3)$ & $214(45.4-1260)$ \\
\hline
\end{tabular}

TABLE 2: UACR comparison between diabetics with and without retinopathy

DR - diabetic retinopathy, UACR - urinary albumin to creatinine ratio

However, on logistic regression analysis, only three variables had an independent association with the presence of DR, namely UACR, known duration of T2DM, and past history of coronary artery disease (Table 3). 


\section{Cureus}

\begin{tabular}{|c|c|c|c|c|}
\hline & \multirow{2}{*}{ Adjusted odds ratio } & \multicolumn{2}{|c|}{$95 \%$ confidence intervals } & \multirow{2}{*}{$p$-value } \\
\hline & & Lower & Upper & \\
\hline Duration of DM & 3.443 & 1.875 & 6.324 & 0 \\
\hline Presence of CAD & 3.955 & 1.562 & 10.016 & 0.004 \\
\hline UACR >30 mg/g & 2.803 & 1.502 & 5.234 & 0.001 \\
\hline
\end{tabular}

TABLE 3: Logistic regression analysis table displaying the variables with statistically significant independent association with the presence of diabetic retinopathy

DM - diabetes mellitus, CAD - coronary artery disease, UACR - urinary albumin to creatinine ratio

185 patients were detected to have albuminuria ( 97 microalbuminuric and 88 microalbuminuria), while the rest (87 patients) were normoalbuminuric at the time of their hospital visit. The mean UACR values were observed to correlate with the increasing severity of DR, with the lowest values of UACR found in those with grade 1 NPDR (171.4[26.5-621.9]) $\mathrm{mg} / \mathrm{g}$ while the set of patients with PDR had the highest UACR $\{1243.4[87.5-2189.25] \mathrm{mg} / \mathrm{g})$.

The ROC curve for UACR in predicting the presence of DR (Figure 1) had an area under the curve of 0.70 (95\% CI 0.638-0.762) (Table 4). On calculating the Youden index, a UACR cut off value of $140 \mathrm{mg} / \mathrm{g}$ was seen to have the best predictability for DR with a sensitivity of $60.5 \%$ and a specificity of $72 \%$, along with positive (PLR) and negative likelihood ratios (NLR) of 2.16 and 0.54 , respectively.

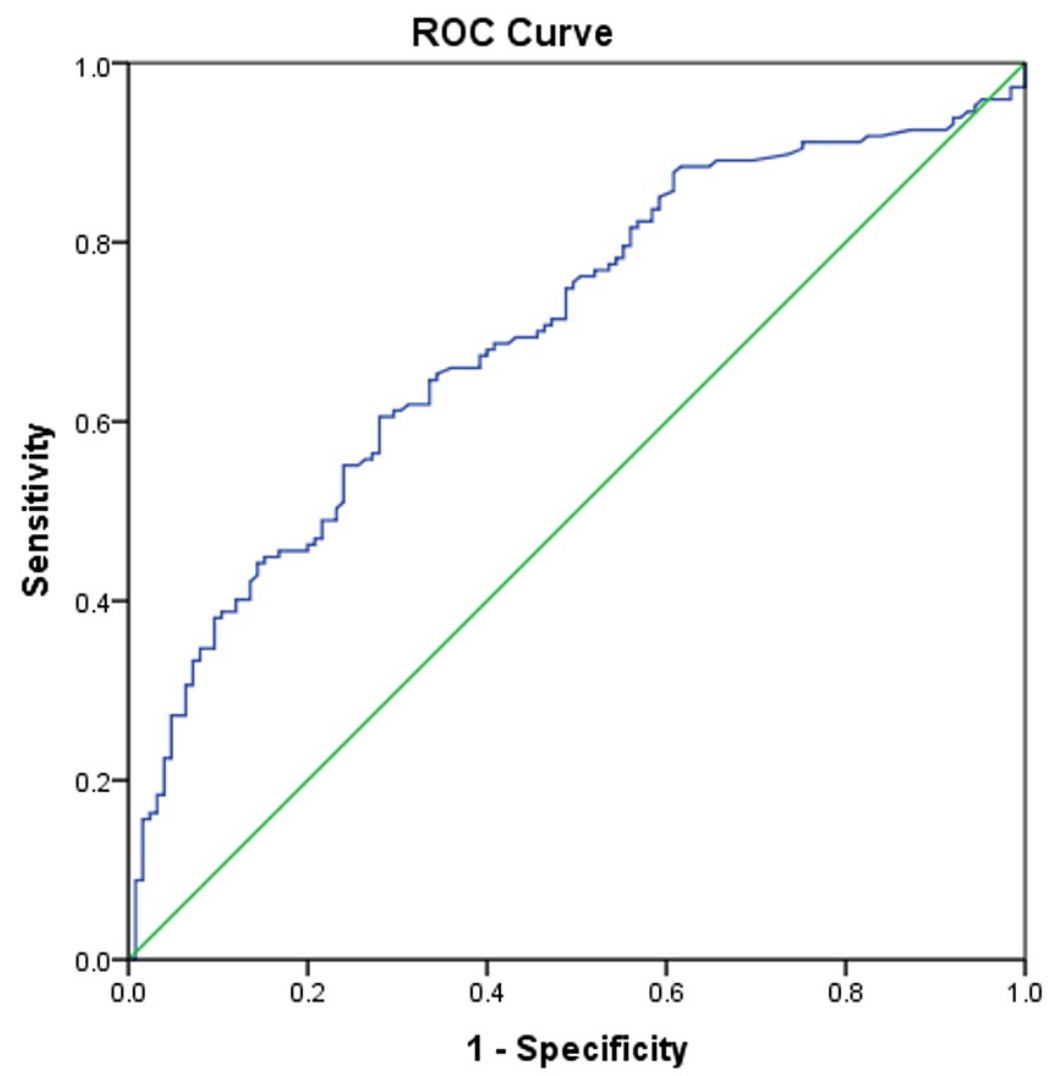

FIGURE 1: Receiver operating characteristic curve for the association of UACR with diabetic retinopathy

$\mathrm{ROC}$ - receiver operating characteristic, UACR - urinary albumin creatinine ratio 


\section{Cureus}

\begin{tabular}{|l|l|l|l|}
\hline Area under the curve & Std. Error & & \multicolumn{2}{c|}{ Asymptotic 95\% confidence interval } \\
Asymptotic Sig.
\end{tabular}

TABLE 4: Area under the receiver operating characteristic curve in Figure 1

Further, the ROC curve was recalculated for the 137 patients in the study who were not on Angiotensinconverting enzyme (ACE) inhibitors and Angiotensin receptor blockers (ARB). The UACR cut-off value of 140 $\mathrm{mg} / \mathrm{g}$ was found to have a comparable sensitivity (60\%), specificity (79\%), PLR (2.65), and NLR (0.24) to indicate the presence of DR in them just as it did for the entire study sample.

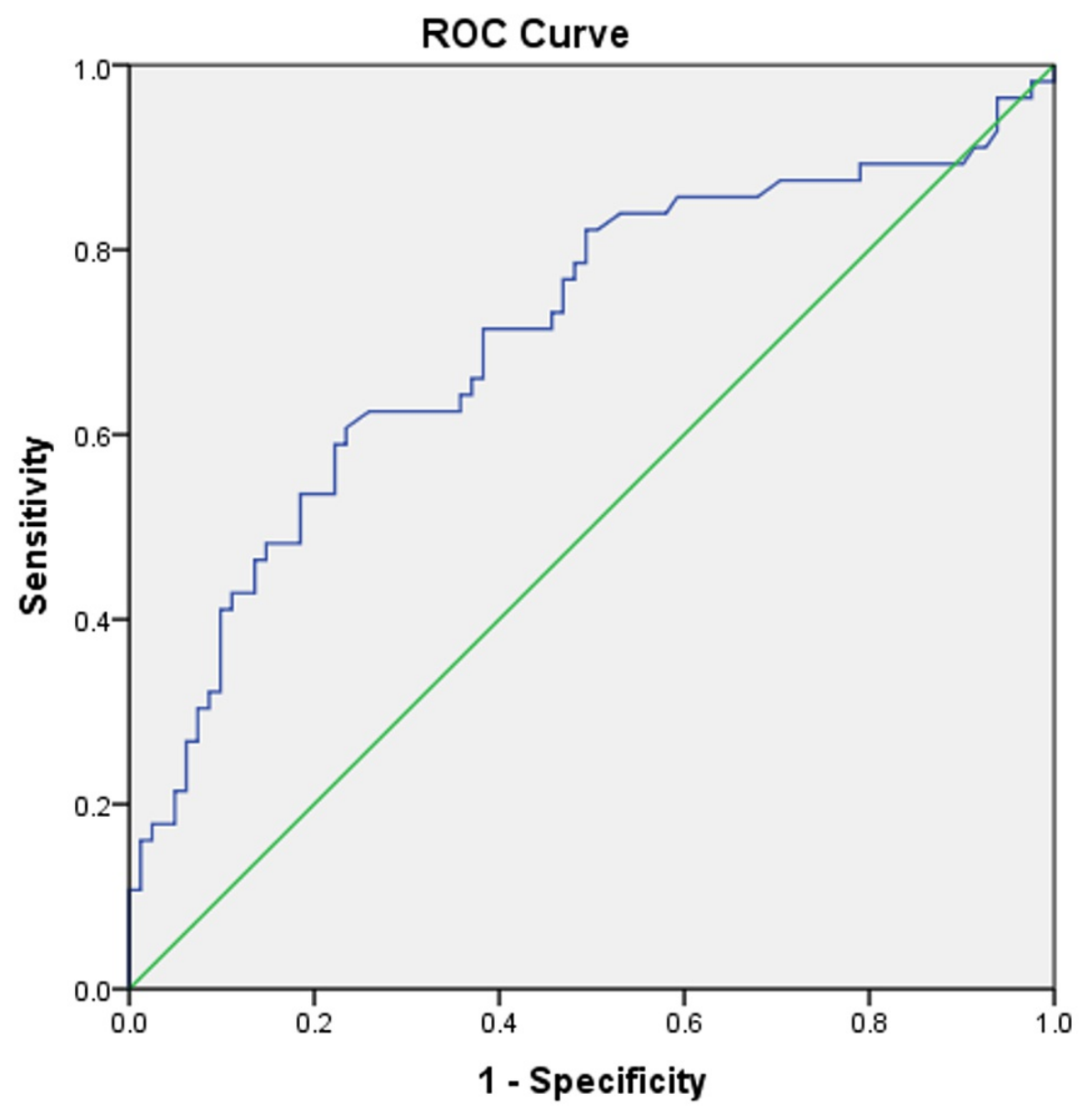

FIGURE 2: Receiver operating characteristic curve for the association of UACR with diabetic retinopathy for those not on ACE inhibitors or ARB

ROC - receiver operating characteristic, UACR - urinary albumin creatinine ratio, ACE - angiotensin-converting enzyme, ARB - angiotensin receptor blockers 


\section{Cureus}

\begin{tabular}{|c|c|c|c|c|}
\hline \multirow{2}{*}{ Area under the curve } & \multirow{2}{*}{ Std. Error ${ }^{a}$} & \multirow{2}{*}{ Asymptotic Sig. ${ }^{\text {b }}$} & \multicolumn{2}{|c|}{ Asymptotic $95 \%$ confidence interval } \\
\hline & & & Lower bound & Upper bound \\
\hline 0.708 & 0.047 & 0.000 & 0.617 & 0.800 \\
\hline
\end{tabular}

TABLE 5: Area under the receiver operating characteristic curve in Figure 2

\section{Discussion}

In the present retrospective study, it was observed that rising levels of UACR were independently associated with the severity of DR among patients with T2DM. Albuminuria was seen to be independently associated with the presence of DR in comparison to normoalbuminuria. UACR beyond $140 \mathrm{mg} / \mathrm{g}$ was observed to be strongly associated with the presence of DR with a sensitivity of $60.5 \%$ and a specificity of $72 \%$. Hence, this suggests that the diabetics with a UACR less than $140 \mathrm{mg} / \mathrm{g}$ may have a lesser probability of having developed DR and may consider postponing their annual fundus examination until the period of peak COVID-19 outbreak passes and COVID-19 related lockdown restrictions are eased. This highlights the potential of UACR to be used as a screening tool to predict the presence of DR. ACE inhibitors and ARB have proven antiproteinuric effects [17]. In order to eliminate the influence of ACE inhibitors and ARB, the data of diabetics without hypertension and CAD was reanalyzed, and it showed that the same cut-off value of $140 \mathrm{mg} / \mathrm{g}$ still performed comparably.

Past studies and systematic reviews have explored the role of albuminuria in predicting the presence of DR with inconsistent results [18-20]. Rani et al. had also demonstrated that patients with micro and macroalbuminuria were two and six times more likely to have DR, respectively, in comparison to those with normal urinary albumin excretion [20]. The cut-off value in our study is higher than those derived in certain other studies $[21,22]$. Potisat et al. failed to show an association between microalbuminuria and DR in their cross-sectional study [23]. But most of these studies have used either dipstick or 24-hour urinary estimation to determine the urinary albumin excretion rate, whereas relatively fewer studies have employed UACR for this purpose $[21,24,25]$. In the present study, although reduced eGFR was seen to be associated with DR on univariate analysis, this association was not evident on multivariate analysis. This is similar to the findings from other studies [26].

The association between DR and UACR is likely to be owing to the common underlying pathophysiological mechanisms involved in their development. Oxidative stress induced by chronic hyperglycemia, formation of advanced glycated end products, activation of protein kinase $\mathrm{C}$ pathway, abnormal activation of the reninangiotensin-aldosterone system may all result in progression of DR and diabetic kidney disease (DKD) in parallel [24]. Endothelial dysfunction is considered to be the pathognomonic feature of the damage that occurs in the microcirculatory system within various organs in the body in T2DM. The extracellular matrix has anionic compounds like heparan sulfate proteoglycan. Enzymes involved in the metabolism of such structural components, such as the $\mathrm{N}$ deacetylase, are altered by hyperglycemia, thereby leading to endothelial dysfunction. Additionally, microalbuminuria and DR have several risk factors in common, including the known duration of diabetes and elevated blood pressure levels. Finally, DR may be accelerated in the presence of elevated fibrinogen and lipoproteins levels due to renal damage [19].

Although several variables in the present study were found to be associated with DR on univariate analysis, only three factors were found to be independently associated with the presence of DR, namely UACR, known duration of diabetes, and past history of CAD. Similar findings have been noted in earlier studies [22,27]. Often the duration of T2DM determined from patients' history does not reflect the true duration of the disease since these patients may not develop symptoms for several years despite the presence of hyperglycemia [28]. T2DM is considered to be the equivalent of atherosclerotic coronary artery disease, yet patients with T2DM may develop silent CAD owing to autonomic dysfunction, which is common in longstanding diabetics. This may delay the detection of CAD [29]. Nevertheless, considering these other two factors in addition to UACR may enhance the effectiveness of DR screening. Developing an algorithm or a formula that incorporates all these parameters together may enhance the precision of DR screening.

Strengths of this study include the employment of robust exclusion criteria and the statistical methods in the analysis. The limitations include its retrospective design, limited study sample size, and that only a single value of UACR was available for all patients. The findings of this study will need to be confirmed using larger prospective trials.

\section{Conclusions}

The presence of albuminuria significantly correlated with the presence of DR in patients with T2DM in this study. During the COVID-19 pandemic, when healthcare services have come under tremendous strain, UACR may be used as a screening tool in the detection of DR. A UACR value of greater than $140 \mathrm{mg} / \mathrm{g}$ may be 
considered as a cut-off to determine the need to undergo fundus examination for DR until the prevailing restrictions due to the pandemic are eased.

\section{Additional Information \\ Disclosures}

Human subjects: Consent was obtained or waived by all participants in this study. Ramaiah Medical College Ethics committee issued approval MSRMC/STD/EC/022/2015. Since this was a retrospective study, ethical clearance was waived. Animal subjects: All authors have confirmed that this study did not involve animal subjects or tissue. Conflicts of interest: In compliance with the ICMJE uniform disclosure form, all authors declare the following: Payment/services info: All authors have declared that no financial support was received from any organization for the submitted work. Financial relationships: All authors have declared that they have no financial relationships at present or within the previous three years with any organizations that might have an interest in the submitted work. Other relationships: All authors have declared that there are no other relationships or activities that could appear to have influenced the submitted work.

\section{Acknowledgements}

Divya Prabhu and Akshay Rao contributed equally to the work and should be considered co-first authors. The authors of this study would like to thank Ms. Radhika K and late Dr. NS Murthy from the department of community medicine at Ramaiah Medical College for their support in performing the statistical analysis for this study.

\section{References}

1. Poddar AK, Khan TA, Sweta K, et al.: Prevalence and causes of avoidable blindness and visual impairment, including the prevalence of diabetic retinopathy in Siwan district of Bihar, India: a population-based survey. Indian J Ophthalmol. 2020, 68:375-80. 10.4103/ijo.IJO_1709_18

2. GBD 2019 Blindness and Vision Impairment Collaborators: Causes of blindness and vision impairment in 2020 and trends over 30 years, and prevalence of avoidable blindness in relation to VISION 2020: the Right to Sight: an analysis for the Global Burden of Disease Study. Lancet Glob Health. 2021, 9:144-60. 10.1016/S2214-109X(20)30489-7

3. Alharbi AM, Alhazmi AM: Prevalence, risk factors, and patient awareness of diabetic retinopathy in Saudi Arabia: a review of the literature. Cureus. 2020, 12:e11991. 10.7759/cureus.11991

4. American Diabetes Association: Microvascular complications and foot care: standards of medical care in diabetes - 2020. Diabetes Care. 2020, 43:S135-51. 10.2337/dc20-S011

5. Chatziralli I, Dimitriou E, Kazantzis D, Machairoudia G, Theodossiadis G, Theodossiadis P: Effect of COVID19-associated lockdown on patients with diabetic retinopathy. Cureus. 2021, 13:e14831. 10.7759/cureus.14831

6. Gupta PC, Kumar MP, Ram J: COVID-19 pandemic from an ophthalmology point of view . Indian J Med Res. 2020, 151:411-8. 10.4103/ijmr.IJMR_1369_20

7. Yokoyama H, Sone H, Oishi M, Kawai K, Fukumoto Y, Kobayashi M: Prevalence of albuminuria and renal insufficiency and associated clinical factors in type 2 diabetes: the Japan Diabetes Clinical Data Management study (JDDM15). Nephrol Dial Transplant. 2009, 24:1212-9. 10.1093/ndt/gfn603

8. Chang $\mathrm{CH}$, Chuang LM: Microalbuminuria and diabetic retinopathy in type 2 diabetic patients: from risk association to risk prediction. J Diabetes Investig. 2013, 4:42-4. 10.1111/jdi.12023

9. Beckman JA, Creager MA: Vascular complications of diabetes. Circ Res. 2016, 118:1771-85. 10.1161/CIRCRESAHA.115.306884

10. Satchell SC, Tooke JE: What is the mechanism of microalbuminuria in diabetes: a role for the glomerular endothelium?. Diabetologia. 2008, 51:714-25. 10.1007/s00125-008-0961-8

11. Witte EC, Lambers Heerspink HJ, de Zeeuw D, Bakker SJ, de Jong PE, Gansevoort R: First morning voids are more reliable than spot urine samples to assess microalbuminuria. J Am Soc Nephrol. 2009, 20:436-43. 10.1681/ASN.2008030292

12. Armstrong T, Bull F: Development of the World Health Organization global physical activity questionnaire (GPAO). J Public Health. 2006, 14:66-70. 10.1007/s10389-006-0024-x

13. Solomon SD, Goldberg MF: ETDRS grading of diabetic retinopathy: still the gold standard? Ophthalmic Res. 2019, 62:190-5. 10.1159/000501372

14. Levey AS, Stevens LA, Schmid CH, et al.: A new equation to estimate glomerular filtration rate. Ann Intern Med. 2009, 150:604-12. 10.7326/0003-4819-150-9-200905050-00006

15. Keane WF, Eknoyan G: Proteinuria, albuminuria, risk, assessment, detection, elimination (PARADE): a position paper of the National Kidney Foundation. Am J Kidney Dis. 1999, 33:1004-10. 10.1016/s02726386(99)70442-7

16. Mandrekar JN: Receiver operating characteristic curve in diagnostic test assessment . J Thorac Oncol. 2010, 5:1315-6. 10.1097/JTO.0b013e3181ec173d

17. Robles NR, Romero B, de Vinuesa EG, Sánchez-Casado E, Cubero JJ: Treatment of proteinuria with lercanidipine associated with renin-angiotensin axis-blocking drugs. Ren Fail. 2010, 32:192-7. 10.3109/08860220903541135

18. Abdelghaffar W, Ghobashy W, Abdo M, El-Baz A, Ibrahim M: Albuminuria as a biomarker for risk of retinopathy in type II diabetic patients in Suez Canal area. Egypt Retina J. 2013, 1:18-22. 10.4103/23475617.135243

19. Aman M, Rasyid H, Sartika SD, et al.: Albumin excretion rate among patients with diabetic retinopathy. 
Caspian J Intern Med. 2020, 11:177-82. 10.22088/cjim.11.2.177

20. Rani PK, Raman R, Gupta A, Pal SS, Kulothungan V, Sharma T: Albuminuria and diabetic retinopathy in type 2 diabetes mellitus Sankara Nethralaya Diabetic Retinopathy Epidemiology And Molecular Genetic Study (SN-DREAMS, report 12). Diabetol Metab Syndr. 2011, 3:9. 10.1186/1758-5996-3-9

21. Lee MK, Han KD, Lee JH, et al.: Normal-to-mildly increased albuminuria predicts the risk for diabetic retinopathy in patients with type 2 diabetes. Sci Rep. 2017, 7:11757. 10.1038/s41598-017-11906-6

22. Ra H, Yoo JH, Ban WH, et al.: Predictors for diabetic retinopathy in normoalbuminuric people with type 2 diabetes mellitus. Diabetol Metab Syndr. 2012, 4:29. 10.1186/1758-5996-4-29

23. Potisat S, Srisubat A, Krairttichai U, Jongsareejit A: The relationship between microalbuminuria by using urine dipsticks and diabetic retinopathy in type 2 diabetes. J Med Assoc Thai. 2008, 91:846-51.

24. Zhuang X, Cao D, Yang D, et al.: Association of diabetic retinopathy and diabetic macular oedema with renal function in southern Chinese patients with type 2 diabetes mellitus: a single-centre observational study. BMJ Open. 2019, 9:e031194. 10.1136/bmjopen-2019-031194

25. Romero-Aroca P, Baget-Bernaldiz M, Navarro-Gil R, et al.: Glomerular filtration rate and/or ratio of urine albumin to creatinine as markers for diabetic retinopathy: a ten-year follow-up study. J Diabetes Res. 2018, 2018:5637130. 10.1155/2018/5637130

26. Seid MA, Akalu Y, Gela YY, et al.: Microvascular complications and its predictors among type 2 diabetes mellitus patients at Dessie town hospitals, Ethiopia. Diabetol Metab Syndr. 2021, 13:86. 10.1186/s13098021-00704-w

27. Cardoso CR, Leite NC, Dib E, Salles GF: Predictors of development and progression of retinopathy in patients with type 2 diabetes: importance of blood pressure parameters. Sci Rep. 2017, 7:4867. 10.1038/s41598-017-05159-6

28. Porta M, Curletto G, Cipullo D, et al.: Estimating the delay between onset and diagnosis of type 2 diabetes from the time course of retinopathy prevalence. Diabetes Care. 2014, 37:1668-74. 10.2337/dc13-2101

29. Fokoua-Maxime CD, Lontchi-Yimagou E, Cheuffa-Karel TE, Tchato-Yann TL, Pierre-Choukem S: Prevalence of asymptomatic or "silent" myocardial ischemia in diabetic patients: protocol for a systematic review and meta-analysis. PLoS One. 2021, 16:e252511. 10.1371/journal.pone.0252511 